\title{
INDEPENDENCE OF A CERTAIN AXIOMATIC SYSTEM
}

\section{LINO GUTIERREZ-NOVOA}

To prove the independence of the system of axioms introduced in [1] we exhibit here ten models, each of them satisfying all the axioms but one; e.g. model $M_{5}$ fails to satisfy $P_{5}$ of [1].

We call model $\Gamma$ the Euclidean 3 -space with the usual vector structure, introduce a suitable order function $\phi$ and the usual notion of orthogonality. Let $A_{i}(\mathrm{i}=1,2,3,4)$ be the position vectors of four points and define:

$$
\phi\left(A_{1}, A_{2}, A_{3}, A_{4}\right)=\text { sign } \operatorname{det}\left|A_{2}-A_{1}, A_{3}-A_{1}, A_{4}-A_{1}\right| .
$$

Model $\Gamma$ shows the relative consistency of the system.

$M_{1}$-In $\Gamma$, take a new order function: $\phi_{1}=|\phi|$.

$M_{2}$ - In $\Gamma$, change the sign of $\phi$ for exactly one nonsingular tetrad and its opposite.

$M_{3}$-Let the $\phi$ of $\Gamma$ be identically 0 .

$M_{4}$-Adjoin one point $X$ to $\Gamma$ and extend $\phi: \phi\left(X, A_{1}, A_{2}, A_{3}\right)$ $=\phi\left(O, A_{1}, A_{2}, A_{3}\right)$ where $O$ is the origin and $A_{i} \in \Gamma$.

$M_{5}$-Vectors of $\Gamma$ with integral components and $\phi$ restricted accordingly.

$M_{6}$ - In the hyperbolic space $H^{3}$ take any orientation function for $\phi$ and keep the usual orthogonality notion.

$M_{T}$-Take the vectors of $\Gamma$ with rational components and restrict $\phi$.

$M_{8}$-Imbed $\Gamma$ in the projective space $P^{3}$ adding the ideal plane $\Omega$. Let $\gamma$ be a real conic on $\Omega$ and define $l \perp \pi$ (in $\Gamma$ ) to mean $l \cap \Omega$ and $\pi \cap \Omega$ are pole and polar with respect to $\gamma$.

$M_{9}$ - Same as before but $l \perp \pi$ is defined only if $l \cap \Omega$ is a twotangent point with respect to $\gamma$.

$M_{10}$-Let $\gamma$ be an elliptic correlation, not a polarity, on $\Omega$, and define $l \perp \pi$ accordingly.

The proof is now complete.

\section{REFERENCE}

1. L. Gutierrez-Novoa, Ten axioms for three-dimensional Euclidean geometry, Proc. Amer. Math. Soc. 19 (1958), 146-152.

UNIVERSITY OF Alabama

Received by the editors May 13, 1968. 